\title{
Long-Lasting Increase of Corticosterone After Fear Memory Reactivation: Anxiolytic Effects and Network Activity Modulation in the Ventral Hippocampus
}

\author{
Anne Albrecht' ${ }^{1,4}$, Gürsel Çalışkan ${ }^{2,4}$, Melly S Oitzl ${ }^{3}$, Uwe Heinemann ${ }^{2}$ and Oliver Stork' \\ IInstitute of Biology, Otto-von-Guericke-University Magdeburg, Magdeburg, Germany; ${ }^{2}$ Institute for Neurophysiology, Charité-Universitätsmedizin \\ Berlin, Berlin, Germany; ${ }^{3}$ SILS-Center for Neuroscience, University of Amsterdam, Amsterdam, The Netherlands
}

\begin{abstract}
Pathological fear and anxiety can be studied, in rodents, with fear conditioning and exposure to reminder cues. These paradigms are thought to critically involve the ventral hippocampus, which also serves as key site of glucocorticoid action in the brain. Here, we demonstrate a long-lasting reduction of kainate-induced gamma oscillations in slice preparations of the ventral hippocampal area CA3, 30 days after a single fear conditioning training. Reduction of gamma power was sensitive to corticosterone application and associated with a decrease in glucocorticoid and mineralocorticoid receptor mRNA expression across strata of the ventral hippocampal CA3. A fear reactivation session $24 \mathrm{~h}$ after the initial conditioning normalized receptor expression levels and attenuated the corticosterone-mediated recovery of gamma oscillations. It moreover increased both baseline and stimulus-induced corticosterone plasma levels and evoked a generalization of fear memory to the background context. Reduced ventral hippocampal gamma oscillation in both fear reactivated and non-reactivated mice were associated with a decrease of anxiety-like behavior in an elevated plus maze. Taking advantage of the circadian fluctuation in corticosterone, we demonstrated the association of high endogenous basal corticosterone plasma concentrations during morning hours with reduced anxiety-like behavior in fear reactivated mice. The anxiolytic effect of the hormone was verified with local applications to the ventral hippocampus. Our data suggest that corticosterone acting on ventral hippocampal network activity has anxiolytic-like effects following fear exposure, highlighting its potential therapeutic value for anxiety disorders. Neuropsychopharmacology (2013) 38, 386-394; doi:I0.1038/npp.2012.192; published online 12 September 2012
\end{abstract}

Keywords: corticosterone; anxiety; fear memory; gamma oscillations; ventral hippocampus

\section{INTRODUCTION}

Altered baseline concentrations of cortisol in blood plasma and dysregulation of the hypothalamus-pituitary-adrenal (HPA) axis response to stress are important features of anxiety disorders (Ströhle and Holsboer, 2003). Recent studies indicate a protective role of elevated cortisol toward anxiety levels, and cortisol or cortisol agonists have been proposed for treatment of anxiety symptoms (Soravia et al, 2006; de Quervain, 2008). However, the relation between cortisol or its equivalent in rodents, corticosterone, and the formation of fear- and anxiety-related symptoms is complex and their neurobiological mechanisms are not well understood (Schwabe et al, 2012).

*Correspondence: Dr A Albrecht, Institute of Biology, Otto-vonGuericke-University Magdeburg, Leipziger Street 44, Magdeburg, 39120, Germany, Tel: +49 (0) 39| 6755 108, Fax: +49 (0) 391 67 55 102, E-mail: anne.albrecht@ovgu.de

${ }^{4}$ These authors contributed equally to this work.

Received 10 May 2012; revised 15 August 2012; accepted 21 August 2012
Classical fear conditioning provides a tool for studying the neurobiology of anxiety disorders such as posttraumatic stress disorder (PTSD) or phobias in rodents (Siegmund and Wotjak, 2006; Stein and Matsunaga, 2006). In this paradigm, a previously neutral stimulus like a tone (conditioned stimulus, CS) is presented together with an aversive stimulus, such as foot shock (unconditioned stimulus, US). The conditioned subject forms an associative memory and will respond to subsequent presentation of the CS or training context with fear in anticipation of the US. During such re-exposure, fear memory is not only retrieved, but the once consolidated memory trace becomes labile and susceptible to modulation, a process called reconsolidation (Alberini, 2011; Rodriguez-Ortiz and Bermúdez-Rattoni, 2007). This process may be utilized to destabilize fear memory in PTSD and phobia patients by updating the reactivated memories with non-fearful information (Schiller et al, 2010). On the other hand, presentation of reminder cues has been used to produce PTSD-like behavioral changes in rodents (Olson et al, 2011).

The ventral hippocampus, through its close interconnection with amygdala and entorhinal cortex (Pitkänen et al, 2000) and its intimate interaction with the HPA stress axis 
(Jacobson and Sapolsky, 1991; Maggio and Segal, 2009), may act as critical mediator of these processes (Bannerman et al, 2004; Trivedi and Coover, 2004). In this study, we addressed the role of the ventral hippocampus in fear reactivation-induced emotional changes, addressing longlasting physiological and molecular changes and their relation to circulating corticosterone levels.

\section{MATERIALS AND METHODS}

Male C57B/6BomTac (M\&B Taconic, Germany) were obtained at an age of 6-7 weeks and maintained in groups of 4-6 on a reversed day-night cycle (lights on at 1900 hours) with food and water ad libitum. Animals were habituated to the environment for 2 weeks before a 6-week test schedule commenced that was varied through five different experiments (see also Supplementary Information, Figure S1). Pretraining anxiety levels were assessed with a light-dark avoidance test (exploration of a brightly lit and the equally sized dark compartment in a single 5-min session). Auditory cued fear conditioning was done as described previously (Laxmi et al, 2003) and $24 \mathrm{~h}$ after training mice were re-exposed to a set of four conditioned fear stimuli in their training context for fear memory reactivation. Long-term effects were tested 4 weeks later by assessing anxiety-like behavior on an elevated plus maze to avoid effects of retesting in the light-dark avoidance test ( $5 \mathrm{~min}$ at low light conditions (10lux); Rehberg et al, 2010). Corticosterone plasma concentrations were determined as described previously (Dalm et al, 2008) via a commercially available 125Icorticosterone radio immunoassay kit. Reactivated fear memory was tested by exposure to four conditioned fear stimuli in their training context. In experiments $1,2,3$, and 5, all tests were performed between 1300 and 1600 hours.

In experiment 1 , the effects of fear memory reactivation on corticosterone plasma levels, anxiety, and fear memory were assessed by comparing the 'reactivation group' $(\mathrm{R} ; N=7)$ receiving the full protocol to a 'no reactivation group' (NR, $N=8$ ), receiving fear conditioning training only and a 'control group' (CTL, $N=8$ ), receiving only tone stimuli but no foot shocks during training.
In experiment 2, kainate-induced gamma oscillation was assessed in ventral hippocampal slices in mice 30 days after fear memory reactivation $(\mathrm{R} ; N=7)$ as well is in animals of the NR $(N=8)$ and CTL $(N=6)$ groups as described before (Liotta et al, 2011).

In experiment 3, mRNA expression of glucocorticoid (GR) and mineralocorticoid receptors (MR) were assessed in sublayers of the ventral hippocampal CA3 region, in groups $\mathrm{R}(N=6)$, NR $(N=6)$, and CTL $(N=6)$.

In experiment 4 , only reactivation training was used, but training and test sessions were either at time point 1 (T1) from 0800 to 0930 hours, $1 \mathrm{~h}$ after lights off when corticosterone is typically high (in an inverse light-dark cycle; Dalm et al, 2005) or at time point 6 (T6) from 1300 to 1500 hours, when corticosterone can be expected to be low. Eight groups were designed ( $N=8$ in each), in which these times were systematically permuted during training, reactivation, and retrieval, allowing for variation of corticosterone through circadian fluctuations. Elevated plus maze test, blood samples collection, and fear memory testing were done at daytimes corresponding to the individual retrieval test.

In experiment 5 , again only mice with reactivation of fear memory were used to assess effects of corticosterone administered to the ventral hippocampus on fear memory and anxiety. In the third week after memory reactivation, guide canulae were implanted in the left and right ventral hippocampi (AP: $-3.08 \mathrm{~mm}$; ML: $\pm 2.9 \mathrm{~mm}$ from Bregma; DV: $2.5 \mathrm{~mm}$ from brain surface; Paxinos and Franklin, 2001), through which either corticosterone (10 ng; $N=9$ ) or vehicle $(N=9)$ was applied in the fourth week after fear conditioning. At $15 \mathrm{~min}$ after drug infusion, anxiety-like behavior was assessed in an elevated plus maze followed by fear memory retrieval $10 \mathrm{~min}$ later.

\section{RESULTS}

\section{Experiment 1: Behavior and Corticosterone are Changed by Fear Conditioning and Fear Reactivation}

Corticosterone. Reactivation of conditioned fear led to enhanced baseline corticosterone plasma concentration

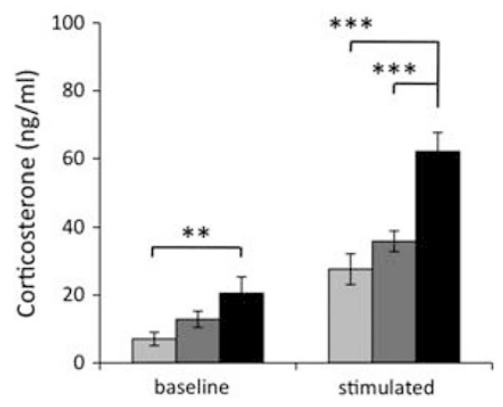

b

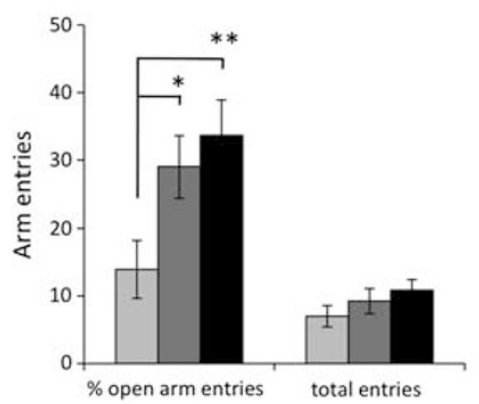

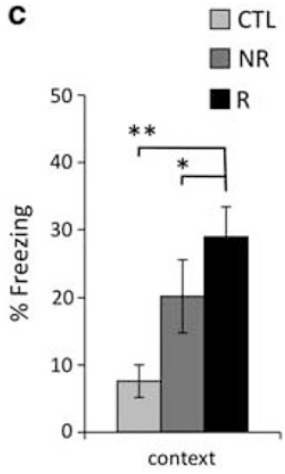

Figure I Fear reactivation elicits long-lasting changes in corticosterone plasma concentration, anxiety, and context fear memory. (a) Corticosterone plasma concentration (CORT) is increased after fear reactivation (R), even under unstimulated conditions. After fear memory testing, the increased corticosterone levels of group $\mathrm{R}$ are maintained at a high level. (b) Anxiety-like behavior in the elevated plus maze is reduced in group R, but also after fear conditioning alone (NR), compared with an unconditioned control group (CTL). The number of total entries to closed and open arms is not altered between groups, suggesting comparable levels of activity in all groups. (c) Fear reactivation increases freezing to the training context. Values are indicated as mean \pm SEM. Significant difference between genotypes with $* p<0.05$; ** $p<0.0$ I; **** $p<0.00$ I. 
(Figure 1a, see also Supplementary Figure S2; ANOVA for group: $\mathrm{F}(2,20)=4.443, p=0.025$; Fisher's LSD post hoc comparison: $p=0.007$ to CTL), whereas fear conditioning training alone was not sufficient $(p=0.119$ to CTL). At $30 \mathrm{~min}$ after fear memory retrieval, corticosterone plasma concentration were further increased $(\mathrm{F}(2,20)=15.924$; $p<0.001)$, again only in group $\mathrm{R}(p<0.001$ to CTL, $p<0.001$ to NR).

Anxiety-like behavior. Pretraining anxiety levels in the light-dark avoidance test were comparable between groups (activity in the light compartment: $\mathrm{F}(2,20)=0.517 ; p=0.604$ ). However, increased open arm entries in the elevated plus maze were observed in both groups, NR (Figure 1b; ANOVA for group: $\mathrm{F}(2,20)=4.86, p=0.019$; Fisher's LSD post hoc comparison: $p=0.03$ to control) and $\mathrm{R}(p=0.008) 4$ weeks post-training. Indicators of general activity and closed arm entries did not differ between groups (total entries: $\mathrm{F}(2,20)=1.279, p=0.295$; distance: $\mathrm{F}(2,20)=2.028, p=0.158$; time active: $\mathrm{F}(2,20)=0.078, p=0.925$; time immobile: $\mathrm{F}(2,20)=0.93, p=0.411$; closed arm entries: $\mathrm{F}(2,20)=$ $0.266, p=0.769)$.

Fear memory. Fear reactivation training increased the freezing response to the training context, (Figure 1c; ANOVA for group: $\mathrm{F}(2,20)=9.197, p=0.001)$ compared with the CTL group $(p<0.001)$ and also with group NR $(p=0.019)$. In contrast, no effect was observed concerning the freezing response to the neutral $(\mathrm{CS}-: \mathrm{F}(2,20)=3.395, p=0.054$; Supplementary Figure S3) and the conditioned acoustic stimuli $(\mathrm{CS}+: \mathrm{F}(2,20)=1.017, p=0.379)$.

In summary, fear conditioning led to anxiolytic-like changes in an elevated plus maze, regardless of its reactivation, but only in the reactivated group enhanced corticosterone and background context memory were observed.

\section{Experiment 2: Fear Reactivation Attenuates the Corticosterone Sensitivity of Fear Conditioning-Induced Changes in Gamma Oscillations}

Normalized gamma power $(20-80 \mathrm{~Hz})$ recorded from the border of stratum lacunosum moleculare and stratum radiatum was significantly decreased (Figures $2 \mathrm{a}$ and b; ANOVA for group: $\mathrm{F}(2,40)=4.42 ; p=0.019$, Fisher's LSD post hoc) in both group NR $(N=14$ slices, seven animals; $0.25 \pm 0.07$, $p=0.010)$ and group $\mathrm{R}(N=15$ slices, seven animals; $0.33 \pm$ $0.07, p=0.017)$ compared with controls $(N=12$ slices, six animals; $1.0 \pm 0.35)$. In parallel recordings from slices of the same animals, preapplication of $1 \mu \mathrm{M}$ corticosterone for 30 min $(N=10-12$ slices per group) attenuated this change and somewhat increased gamma power in both NR (paired comparison: $0.25 \pm 0.07$ vs $0.77 \pm 0.31, p=0.061$ ), but less in $\mathrm{R}$ (paired comparison: $0.33 \pm 0.07$ vs $0.50 \pm 0.14, p=0.31$ )
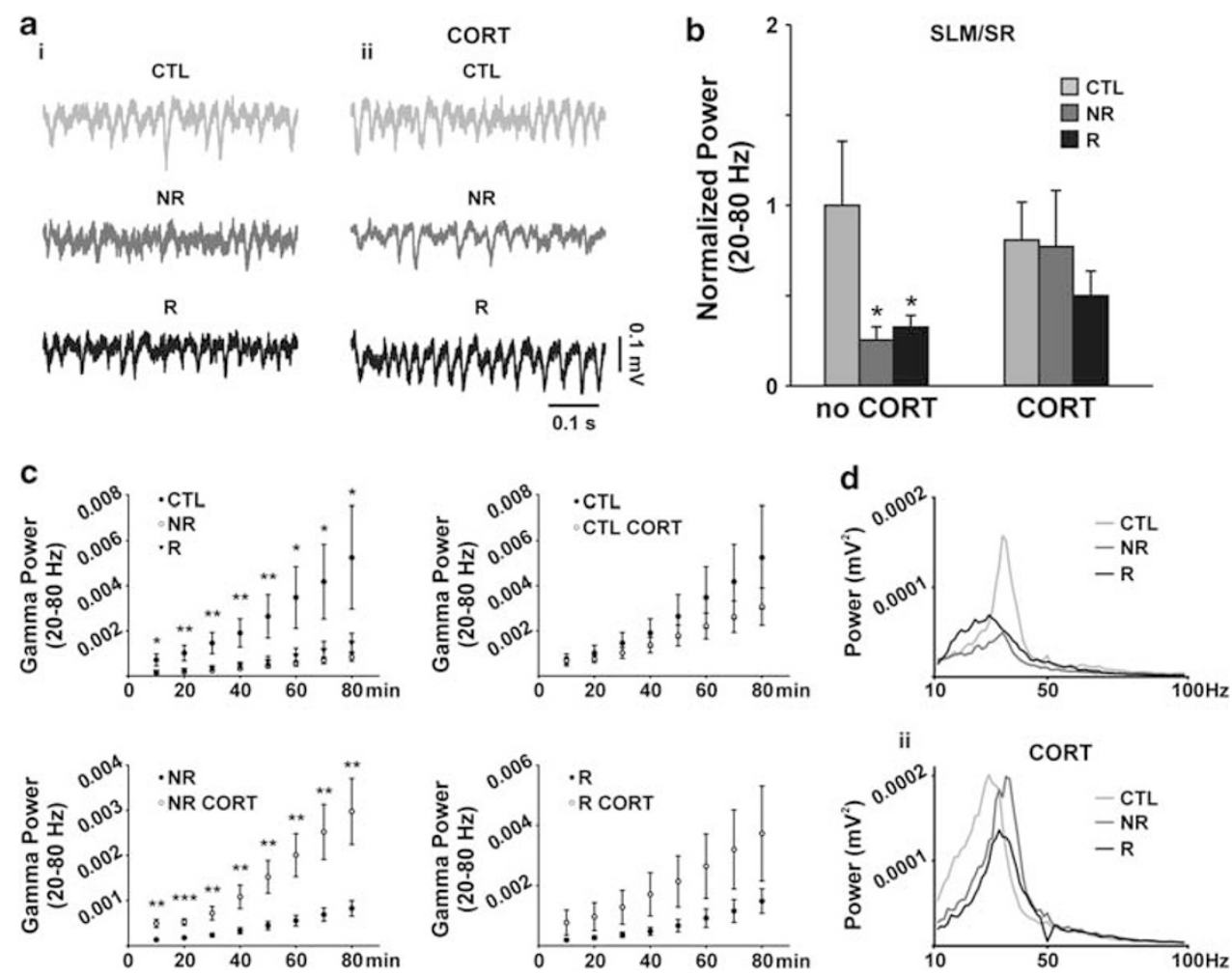

Figure 2 Fear conditioning and its reactivation reduce kainate-induced gamma oscillations in ventral hippocampal slices ex vivo. (a) Example traces of gamma oscillations from stratum radiatum (SR)/stratum lacunosum moleculare (SLM) (i) without corticosterone (no CORT) and (ii) with corticosterone application (I $\mu \mathrm{M} ; \mathrm{CORT}$ ) in unconditioned controls (CTL), non-reactivated (NR), and fear reactivated (R) animals. (b) Summary graph showing the normalized gamma power $(20-80 \mathrm{~Hz})$ recorded from the border of SR and SLM reveals that both fear conditioning and reactivation reduce gamma power. (c) Summary graphs show the development of gamma oscillation power. Note the reduced gamma power in slices from groups NR and R compared with unconditioned controls. Preapplication of corticosterone has pronounced effect on gamma power in NR slices, but not in CTL or R slices. (d) Examples of power spectra from slices (i) without and (ii) with application of I $\mu \mathrm{M}$ corticosterone (CORT). Values are indicated as mean \pm SEM. Significant difference between groups with $* p<0.05$; ** $p<0.01$; **** $p<0.00$ I. 
and not in CTL (paired comparison: $1.0 \pm 0.35$ vs $0.81 \pm$ $0.21, p=0.95)$.

In a third subset of slices from the same animals $(N=5-7$ slices per group), continuous 80 -min recordings were performed to determine the development of kainate-induced gamma oscillations (Figure 2c). The increase in gamma power was significantly lower already after 10 min of kainate wash-in (ANOVA for group: $\mathrm{F}(2,20)=4.83$; $p=0.022$, Fisher's LSD post hoc) in both NR $\left(0.00013 \pm 0.00005 \mathrm{mV}^{2}\right.$, $p=0.011)$ and $\mathrm{R}\left(0.00019 \pm 0.00010 \mathrm{mV}^{2}, p=0.020\right)$ compared with CTL $\left(0.00071 \pm 0.00027 \mathrm{mV}^{2}\right)$. After $80 \mathrm{~min}$, the gamma power was still significantly diminished in NR and $\mathrm{R}$ (ANOVA for group: $\mathrm{F}(2,20)=3.71 ; p=0.046$, Fisher's LSD post hoc). Slices of group NR preapplied with $1 \mu \mathrm{M}$ corticosterone showed a faster development of gamma oscillations compared with slices without corticosterone (paired comparison: after $20 \mathrm{~min}, 0.00018 \pm 0.00003$ vs $0.00053 \pm 0.00007, p=0.0001$; after $80 \mathrm{~min}, 0.0008 \pm 0.00017 v s$ $0.0030 \pm 0.00073, p=0.005)$. In slices from group $\mathrm{R}$ animals and in CTL slices, corticosterone had no significant effect on gamma development (Figure 2c). Auto-correlation analysis confirmed the reduction of gamma oscillations after fear conditioning and its reactivation, but only nonsignificant recovery was observed in group NR after application of corticosterone. No change of gamma peak frequency was observed (Supplementary Figure S4).

\section{Experiment 3: Conditioned, but Not Reactivated Fear Leads to Reduced GR and MR Expression}

Assessment of mRNA levels with quantitative PCR revealed a reduction of GR and $M R$ expression across the strata of ventral hippocampal CA3, but only if conditioned fear was not reactivated. A three-way ANOVA for gene (GR/MR), stratum (SR/SP/SO), and group (R/NR/CTL) revealed strong impact of each factor on expression levels (Figure 3; gene: $(\mathrm{F}(2,89)=22.347, \quad p=0.000 ;$ stratum: $\mathrm{F}(2,89)=102.948$, $p=0.000$; group: $\mathrm{F}(2,89)=5.344, p=0.006)$. Moreover, expression differences between GR and MR were evident that were dependent on the different strata (interaction of stratum and gene: $\mathrm{F}(2,89)=39.345 ; p=0.000$; stratum effect for GR: $\mathrm{F}(2,45)=93.408 ; p=0.000$; for $\mathrm{MR}: \mathrm{F}(2,45)=$ $13.208 ; p=0.000)$. Post hoc comparison of the group effects revealed a significant downregulation of GR and $M R$ in group NR (Fisher's LSD $p=0.005$ compared with CTL), but not in group $\mathrm{R}(p=0.649$ compared with $\mathrm{CTL} ; p=0.018$ compared with NR).

\section{Experiment 4: Endogenous Corticosterone Levels in the Fear Reactivation Group Negatively Correlate with the Anxiety-Like Behavior}

Hormonal rhythmicity. A circadian fluctuation of corticosterone plasma concentrations was maintained after fear reactivation, as we found high levels $1 \mathrm{~h}$ after the beginning of the dark phase (T1) and lower concentration $5 \mathrm{~h}$ later (T6), both under unstimulated conditions (Supplementary Figure S5A; ANOVA for time of testing: $F(1,47)=38.536$; $p<0.001$ ) and after fear memory retrieval (ANOVA: $\mathrm{F}(1,47)=18.186 ; p<0.001)$. Enhanced baseline levels occluded the stress-induced response at T1 (repeated-measure ANOVA with corticosterone concentration before and after stimulation $\mathrm{F}(1,24)=3.961 ; p=0.058)$ whereas significant increase on testing was detected at $\mathrm{T} 6(\mathrm{~F}(1,23)=90.796$; $p<0.001)$. No interaction was observed of testing time with training time (CORT base: $\mathrm{F}(1,45)=2.280, p=0.138$; CORT stim: $\mathrm{F}(1,45)=0.181, p=0.673)$.

Daytime effects on anxiety. At T1, animals displayed more entries into the open arm of the elevated plus maze than at T6 (Supplementary Figure S5B; ANOVA for time of testing: $\mathrm{F}(1,47)=6.339 ; p=0.015)$ without differences in general activity (number of total arm entries: $\mathrm{F}(1,47)=1.74 ; p=0.194$; distance: $\mathrm{F}(1,47)=1.226 ; p=0.274$; time active: $\mathrm{F}(1,47)=$ $0.242 ; p=0.625)$. This pattern was inverse to anxiety-like

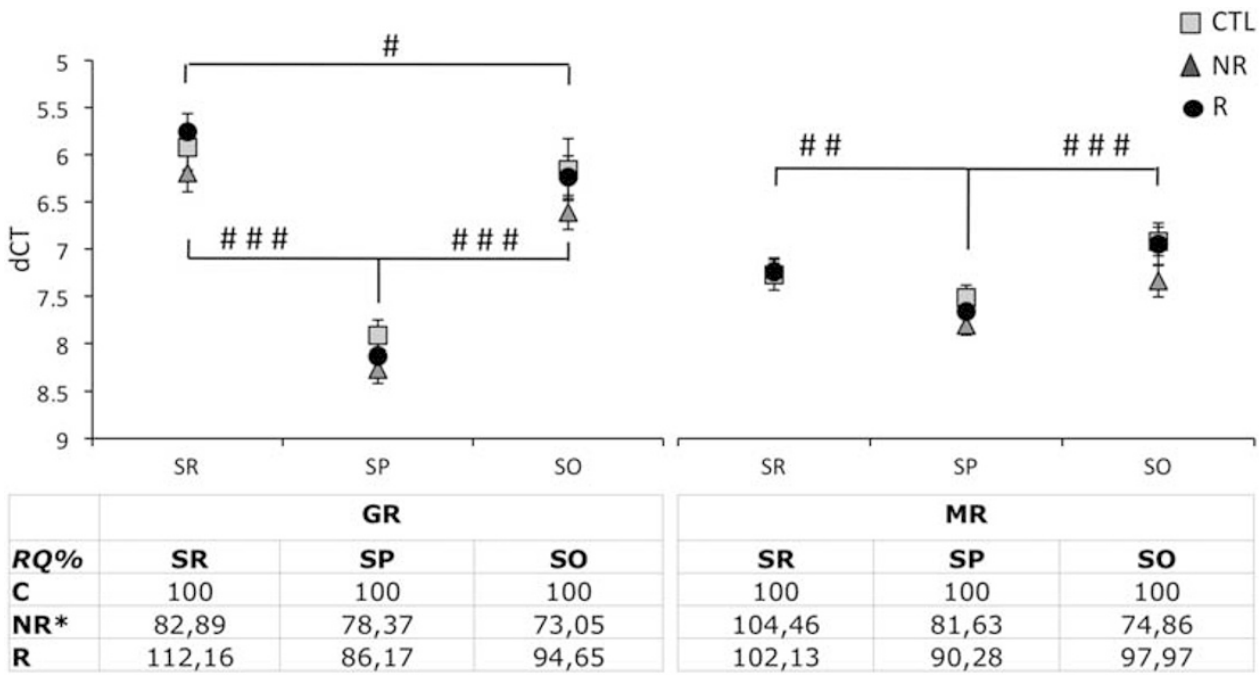

Figure 3 Expression of GR and MR mRNA is reduced in the ventral hippocampal CA3 after fear conditioning. Expression normalized to the internal control gene GAPDH (dCT values) revealed differential expression of GR and MR in stratum radiatum (SR), stratum pyramidale (SP), and stratum oriens (SO) of the ventral CA3 for all groups. The overall expression of both genes was reduced in group NR, but not in group R. Values are mean dCT \pm SEM. For quantification of behaviorally induced expression changes refer to the table below graph (relative quantification with CTL expression levels set to I00\%). Significant difference between groups with ${ }^{*} p<0.05$; significant difference between CA3 sublayers with ${ }^{\#} p<0.05$; ${ }^{\# \#} p<0.01$; ${ }^{\# \#} p<0.00$ I. 
behavior in the pretraining light-dark avoidance test, where activity (ANOVA for time of testing: $\mathrm{F}(1,47)=10.504 ; p=$ $0.002)$ and time spent in the light compartment $(F(1,47)=$ $10.032 ; p=0.003$ ) were increased at T6 (Supplementary Figure S5C).

Daytime effects on fear memory. A high contextual response was observed in all groups, which was neither generally affected by the time of training $(\mathrm{F}(1,47)=1.649 ; p=0.205)$, nor by the time of reactivation $(\mathrm{F}(1,47)=0.524 ; p=0.473)$ or retrieval $(\mathrm{F}(1,47)=3.836 ; p=0.056)$.

High vs low post-reactivation corticosterone. Anxiety levels were low when corticosterone concentrations were high, at $\mathrm{T} 1$, and the percentage of open arm entries was correlated with basal corticosterone concentrations and over all time points (Pearson's correlation coefficient 0.267, $p=0.032$ ). As frequency analysis revealed strong interindividual differences and overlapping distribution of unstimulated corticosterone plasma concentrations at $\mathrm{T} 1$ and T6 (Figure 4a), we reanalyzed our data with respect to basal corticosterone levels. Groups were defined in relation to the median corticosterone concentration $(57.81 \mathrm{ng} / \mathrm{ml})$. High and low post-reactivation corticosterone groups differed almost fourfold in unstimulated corticosterone plasma concentrations (mean \pm SEM: $25.08 \pm 2.6 \mathrm{ng} / \mathrm{ml}$ in low $v s 94.34 \pm 6.42 \mathrm{ng} / \mathrm{ml}$ in high post-reactivation corticosterone; ANOVA: $\mathrm{F}(1,47)=96.912, p<0.001)$. The difference was maintained, although less pronounced after memory retrieval $(85.79 \pm 6.05 \mathrm{ng} / \mathrm{ml}$ in low $v s 100.55 \pm 3.89 \mathrm{ng} / \mathrm{ml}$ in high post-reactivation corticosterone; ANOVA: $\mathrm{F}(1,47)=$ $4.278, p<0.044)$. Within-group comparison showed that the corticosterone response to retrieval was diminished in animals with high basal corticosterone (Figure $4 \mathrm{~b}$; repeatedmeasure ANOVA: $\mathrm{F}(1,24)=0.870, p=0.360)$, whereas animals with low basal corticosterone increased levels significantly on retrieval $(\mathrm{F}(1,23)=156.523 ; p<0.001)$.

Relation to anxiety. The high post-reactivation corticosterone group showed increased open arm entries in the elevated plus maze (Figure 4c; mean \pm SEM: $27.672 .93 \%$ vs $17.4 \pm 3.79 \%$; ANOVA for group effect: $F(1,47)=4.65$; $p=0.036$ ), thus indicating decreased anxiety levels.

Effect on fear memory. ANOVA revealed an interaction of post-reactivation corticosterone group and reactivation daytime on contextual freezing (Figure $4 \mathrm{~d} ; \mathrm{F}(1,45)=4.264$; $p=0.045$ ). Paired comparison demonstrates decreased freezing in the low post-reactivation corticosterone group when fear memory had been reactivated $1 \mathrm{~h}$ after lights off at T1 (ANOVA for effect of retrieval time in low responders: $\mathrm{F}(1,22)=5.986 ; p=0.023)$, but not $5 \mathrm{~h}$ later at $\mathrm{T} 6(\mathrm{~F}(1,23)=$
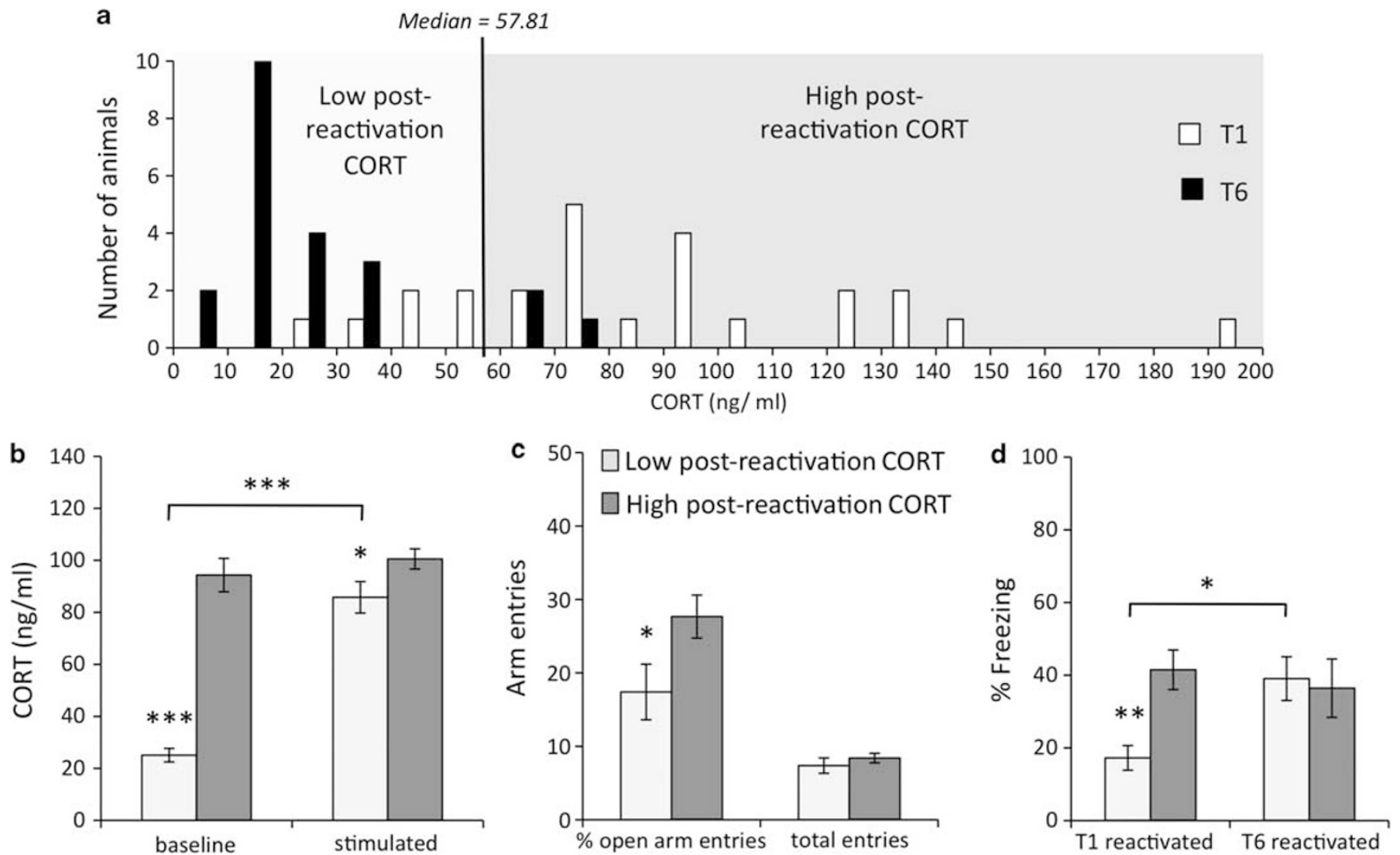

Figure 4 High levels of post-reactivation corticosterone correspond with low anxiety but contextual generalization. (a) Histogram showing the distribution of individual basal corticosterone plasma concentration at I (TI) and $6 \mathrm{~h}(\mathrm{~T} 6)$ after lights off. Based on the median (57.8I ng/ml), mice were assigned to a low and a high post-reactivation corticosterone group. (b) The high post-reactivation corticosterone group shows near maximal plasma concentrations of corticosterone (CORT) under unstimulated conditions and fails to significantly increase on fear memory retrieval. (c) The high postreactivation corticosterone group shows reduced anxiety-like behavior in the elevated plus maze. (d) The low post-reactivation corticosterone group expresses reduced freezing toward the background context if fear reactivation is done at TI, indicating a state-specific memory effect. Values are indicated as mean \pm SEM. Significant difference between groups with $* p<0.05$; $* * 0.01$; $* * * * 0.00$ I. 
$0.068 ; p=0.796)$. In contrast, in the high post-reactivation corticosterone group contextual freezing was high at $\mathrm{T} 1$ as well as at T6 (paired comparison: ANOVA for corticosterone group effect at T1: $\mathrm{F}(1,22)=8.876, p=0.007$; ANOVA for effects of reactivation time in high post-reactivation corticosterone group: $\mathrm{F}(1,23)=0.288 ; p=0.597)$.

\section{Experiment 5: Infusion of Corticosterone to the Ventral Hippocampus Reduces Anxiety-Like Behavior in Fear Reactivated Mice}

Corticosterone injections into the ventral hippocampus increased the exploration of open arms in the elevated plus maze compared with vehicle injected controls (Student's t-test: $\mathrm{T}(16)=3.153 ; p=0.006)$, without affecting the total number of arm entries as a measure of general activity $(\mathrm{T}(16)=0.487 ; p=0.633$; Figure $5 \mathrm{a})$. In contrast, the local corticosterone application had no effect on the freezing behavior displayed in the background context $(\mathrm{T}(16)=$ $0.025 ; p=0.980$, Figure $5 \mathrm{~b})$, or in response to a CS $(\mathrm{T}(16)=1.693 ; p=0.111)$ or to the $\mathrm{CS}+(\mathrm{T}(16)=1.210$; $p=0.244)$.

\section{DISCUSSION}

In this study, we show that the reactivation of fear memory with a reminder cue in mice is associated with long-lasting changes in endogenous corticosterone levels and corticosterone sensitivity of ventral hippocampal gamma oscillations. High morning levels of endogenous corticosterone and corticosterone application to the ventral hippocampus reduced anxiety-like behavior in fear reactivated mice. These data are in line with a potential therapeutic value of corticosteroids in anxiety disorder patients (Schelling et al, 2004; Soravia et al, 2006; de Quervain, 2008) and implicate the ventral hippocampus in these processes.

Remarkably, we observed an increase in exploration of the aversive open arms of an elevated plus maze in both fear conditioned and fear reactivated mice. Similar anxiolysis has previously been shown after chronic mild stress (D'Aquila et al, 1994) and after highly aversive context conditioning (Radulovic et al, 1998; Laxmi et al, 2003). The ventral
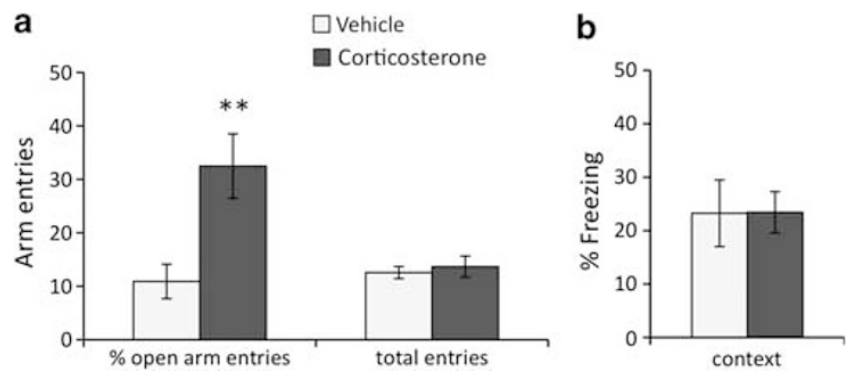

Figure 5 Local administration of corticosterone to the ventral hippocampus decreases anxiety-like behavior in fear reactivated mice. (a) Corticosterone $(10 \mathrm{ng})$ was injected into the ventral hippocampus of fear reactivated mice on $\mathrm{T} 6$, that is, with low endogenous levels, leading to a reduced anxiety-like behavior in the elevated plus maze compared with vehicle-injected controls. (b) Local corticosterone application has no influence on background context generalization. Values are indicated as mean \pm SEM. Significant difference between groups with $* * p<0.01$. hippocampus is critically involved in mediating anxietyrelated behavior in the plus maze task, with lesions of the structure having an anxiolytic-like effect (Kjelstrup et al, 2002; Bannerman et al, 2004). In addition, the ventral hippocampus supports also acquisition and expression of auditory and contextually conditioned fear (Bannermann et al, 2004; Maren and Holt, 2004; Trivedi and Coover, 2004; Rudy and Matus-Amat, 2005). We therefore postulated an involvement of the ventral hippocampus in the observed behavioral changes.

Indeed, we found that fear conditioning results in a significant reduction in the power of kainate-induced gamma oscillations in slice preparations of the ventral hippocampus. Hippocampal gamma $(30-80 \mathrm{~Hz})$ oscillations, which emerge from rhythmic activity of GABAergic interneurons (Buzsáki 2001; Gloveli et al, 2005), shape information flow in the hippocampus and interconnected limbic areas associated with the encoding and retrieval of memory (Hájos and Paulsen 2009; Montgomery and Buzsáki 2007). Kainateinduced gamma oscillations reflect levels of gamma oscillations in vivo and have been correlated with learned avoidance behavior ( $\mathrm{Lu}$ et $\mathrm{al}, 2011)$ and the avoidance of open segments of a zero maze in Clock mutant mice (Dzirasa et al, 2011). Thus, our data are in good agreement with an involvement of gamma oscillations in the ventral hippocampus in anxiety and/or avoidance behavior.

Neural activity in the ventral hippocampus has profound impact on the regulation of the HPA axis (Herman et al, 1995) and in turn is highly sensitive to corticosterone (Maggio and Segal, 2007; 2009). In fact, we could restore the power of gamma oscillations in slice preparations from fear conditioned animals through corticosterone application. The recovery was selective with respect to behavioral state, as corticosterone did not affect gamma power in unconditioned controls. This is in line with previous observations in slices from naive mice, where corticosterone reduced the frequency regularity of carbachol-induced gamma oscillations but did not affect gamma peak power or overall power (Weiss et al, 2008). Considering the observed reduction of GR and MR mRNA expression across CA3 strata and the unaltered plasma corticosterone levels of fear conditioned animals, it thus appears that a single fear conditioning session was sufficient to induce local changes of corticosterone-sensitive cellular functions in the ventral hippocampus. This is in line with a stress-induced 'tuning' of ventral hippocampus network activity and GABAergic function reported previously (Orchinik et al, 2001; Maggio and Segal, 2009), although such persistent changes have not previously been reported in this region following fear conditioning.

On the other hand, exposure to reminder cues has been shown to induce lasting PTSD-related dysfunction following fear conditioning in mice (Olson et al, 2011). When reactivated, fear memory becomes modified and can either be reconsolidated (eg, following a single exposure) or extinguished (eg, following repetitive exposures). Both processes have been discussed as targets for the treatment of excessive fear (Quirk et al, 2010; Schiller et al, 2010). In our experiments, mice were re-exposed to a single reactivation session with a set of four non-reinforced CS + , which allows for fear memory reconsolidation but not fear extinction (Laxmi et al, 2003; Rehberg et al, 2010). Such reconsolidation is thought to reflect an updating process, where memory 
enters a labile phase that partly resembles the original memory consolidation and then is restored in a slightly modified form (Alberini, 2011; Rodriguez-Ortiz and Bermúdez-Rattoni 2007). Like the primary fear development, reconsolidation appears to be critically dependent on glucocorticoids (Wang et al, 2008; Blundell et al, 2011).

Our data also suggest a lasting modification of corticosterone-sensitive cellular functions in the ventral hippocampus. After reactivation of fear memory, anxiety-like behavior and gamma oscillations were maintained at reduced levels, comparable to those after fear conditioning alone. However, we observed a profound loss of corticosterone sensitivity of the network activity and a return of GR and MR mRNA expression to control levels. At the same time, the fear reactivation group showed an enhancement of baseline and stimulus-induced corticosterone levels, potentially through a disturbance of ventral hippocampus-mediated feedback inhibition of the HPA axis. Moreover, freezing to the background context but not the auditory cue was increased after fear memory reactivation, in agreement with our previous observations (Rehberg et al, 2010). Evidence suggests that the formation, retrieval, and reconsolidation of fear memory are dependent on corticosterone; however, effects are strictly dependent on the time point of intervention. Although corticosterone administration shortly before training improves fear conditioning through activation of GR (McGaugh, 2004; Schwabe et al, 2012), corticosterone administration before and inhibition of GR shortly after retrieval impair reconsolidation of fear memory (Cai et al, 2006). Moreover, post-retrieval application reduced freezing to the context only in animals trained with high but not with moderate shock intensity (Abrari et al, 2008). Hence, depending on the tuning of fear memory-related brain circuits by different training conditions corticosterone can facilitate or can attenuate conditioned fear. Genetic and experience-dependent differences in HPA axis regulation are therefore likely to disposition for the individual responding, which may be reflected in the individual variability of endogenous corticosterone levels in our experiments (Figure 4).

Strikingly, after fear memory reactivation, the sensitivity of gamma oscillations to corticosterone was lost although levels of GR and MR expression were comparable to those in naive mice. The cellular change may reflect an adaptive response to enhanced levels of the hormone, and may protect from hippocampal structural and functional impairments (Stranahan et al, 2008), which are frequently observed after prolonged increase in baseline corticosterone (Conrad 2006). On the other hand, enhanced corticosterone levels could serve to compensate for the reduced corticosterone sensitivity in the ventral hippocampus and to maintain low anxiety levels in the reactivated mice, like those observed after fear conditioning per se. In addition, they might be responsible for the observed contextual generalization of the fear memory acting, for example, via the dorsal hippocampus (Kaouane et al, 2012). Therefore, we further analyzed the impact of corticosterone on anxiety-related behavior in fear reactivated animals.

Hippocampal feedback controls the strength and duration of stress-induced corticosterone secretion, but also the circadian fluctuation of the hormone (Jacobson and Sapolsky, 1991). However, we found circadian fluctuations of corticosterone concentrations to be maintained after fear memory reactivation, with levels that match other observations in C57Bl/6J mice (Dalm et al, 2005). By permutating the daytime of training, memory reactivation and testing, we could thus investigate potential effects of endogenous corticosterone levels on fear and anxiety. Indeed, we found that higher corticosterone concentrations at the peak of the circadian rhythm, $1 \mathrm{~h}$ after lights off (T1), were associated with lower levels of anxiety-like behavior than those measured on $5 \mathrm{~h}$ later (T6; Supplementary Figures S5A, C). This could be further confirmed by analyzing the behavior of animals solely on the basis of their basal corticosterone concentrations, and regardless of daytime. Animals with low endogenous corticosterone displayed different contextual fear memory according to the daytime of previous memory reactivation, thus likely reflecting a state-dependent memory effect. This effect, however, was not seen under high endogenous corticosterone, which was generally associated with strong contextual generalization (Figure 4d).

To directly test the function of corticosterone in the ventral hippocampus, we finally applied corticosterone locally at T6, that is, in a period of low endogenous levels. This led to a profound increase of open arm exploration in the plus maze, confirming the anxiolytic-like effect of corticosterone, and supporting the role of the ventral hippocampus therein. At the same time, ventral hippocampal corticosterone injections failed to enhance the contextual response, although administration at the same dose to the dorsal hippocampus has been shown to increase contextual fear memory and to evoke contextual generalization (Kaouane et al, 2012). Anxiolytic effects of acute corticosterone have been reported $>30$-year ago (File et al, 1979), but most studies report anxiogenic-like effects after single or chronic application (eg, Mitra and Sapolsky, 2008). Our data show that the ventral hippocampus mediates anxiolytic effects of corticosterone after fear reactivation.

Evidence for beneficial effects of elevated glucocorticoid levels toward anxiety states also comes from patient studies. Panic disorder patients with high cortisol release during attacks display a better outcome of exposure therapy (Siegmund et al, 2011). In phobic patients, cortisol release during exposure to the phobic stimulus can buffer fear symptoms and cortisone administration before exposure decreases subjective feelings of fear (Soravia et al, 2006). Even in patients that already have established chronic PTSD, daily administration of low-dose cortisol is sufficient to reduce symptoms of traumatic memories (reliving, nightmares; de Quervain, 2008). Rhythmic network activities in the ventral hippocampus and their experience-dependent change may have a critical role in these processes.

\section{ACKNOWLEDGEMENTS}

We are grateful to $\mathrm{C}$ Obst and $\mathrm{F}$ Webers for excellent technical assistance and to A Deter and T Nawrath for animal care. The work was supported by grants from the German Israeli Project Cooperation (DIP) to UH and OS, the Collaborative Research Program SFB779 to OS, by the excellence cluster NeuroCure to UH, by Eurocores 07EuroSTRESS-FP-005 to MSO and by the DFG graduate 
college GRK 1123 'Cellular mechanisms of learning and memory consolidation' to GC.

\section{DISCLOSURE}

The authors declare no conflict of interest.

\section{REFERENCES}

Abrari K, Rashidy-Pour A, Semnanian S, Fathollahi Y (2008). Administration of corticosterone after memory reactivation disrupts subsequent retrieval of a contextual conditioned fear memory: dependence upon training intensity. Neurobiol Learn Mem 89: 178-184.

Alberini CM (2011). The role of reconsolidation and the dynamic process of long-term memory formation and storage. Front Behav Neurosci 5: 12.

Bannerman DM, Rawlins JN, McHugh SB, Deacon RM, Yee BK, Bast $T$ et al (2004). Regional dissociations within the hippocampusmemory and anxiety. Neurosci Biobehav Rev 28: 273-283.

Blundell J, Blaiss CA, Lagace DC, Eisch AJ, Powell CM (2011). Block of glucocorticoid synthesis during re-activation inhibits extinction of an established fear memory. Neurobiol Learn Mem 95: 453-460.

Buzsáki G (2001). Hippocampal GABAergic interneurons: a physiological perspective. Neurochem Res 26: 899-905.

Cai WH, Blundell J, Han J, Greene RW, Powell CM (2006). Postreactivation glucocorticoids impair recall of established fear memory. J Neurosci 26: 9560-9566.

Conrad CD (2006). What is the functional significance of chronic stress-induced CA3 dendritic retraction within the hippocampus? Behav Cogn Neurosci Rev 5: 41-60.

Dalm S, Enthoven L, Meijer OC, van der Mark MH, Karssen AM, de Kloet ER et al (2005). Age-related changes in hypothalamicpituitary-adrenal axis activity of male C57BL/6J mice. Neuroendocrinology 81: 372-380.

Dalm S, Brinks V, van der Mark MH, de Kloet ER, Oitzl MS (2008). Non-invasive stress-free application of glucocorticoid ligands in mice. J Neurosci Methods 170: 77-84.

D'Aquila PS, Brain P, Willner P (1994). Effects of chronic mild stress on performance in behavioural tests relevant to anxiety and depression. Physiol Behav 56: 861-867.

de Quervain DJ (2008). Glucocorticoid-induced reduction of traumatic memories: implications for the treatment of PTSD. Prog Brain Res 167: 239-247.

Dzirasa K, McGarity DL, Bhattacharya A, Kumar S, Takahashi JS, Dunson D et al (2011). Impaired limbic gamma oscillatory activity synchrony during anxiety-related behaviour in a genetic model mouse model of bipolar mania. J Neurosci 31: 6449-6456.

File SE, Vellucci SV, Wendlandt S (1979). Corticosterone - an anxiogenic or an anxiolytic agent? J Pharm Pharmacol 31: 300-305.

Furlan PM, DeMartinis N, Schweizer E, Rickels K, Lucki I (2001). Abnormal salivary cortisol levels in social phobic patients in response to acute psychological but not physical stress. Biol Psychiatry 50: 254-259.

Gloveli T, Dugladze T, Rotstein HG, Traub RD, Monyer H, Heinemann U et al (2005). Orthogonal arrangement of rhythmgenerating microcircuits in the hippocampus. Proc Natl Acad Sci USA 102: 13295-13300.

Hájos N, Paulsen O (2009). Network mechanisms of gamma oscillations in the CA3 region of the hippocampus. Neural Net 22: $1113-1119$.

Herman JP, Cullinan WE, Morano MI, Akil H, Watson SJ (1995). Contribution of the ventral subiculum to inhibitory regulation of hypothalamo-pituitary-adrenocortical axis. J Neuroendocronol 7: 475-482.
Jacobson L, Sapolsky R (1991). The role of the hippocampus in feedback regulation of the hypothalamic-pituitary-adrenocortical axis. Endocr Rev 12: 118-134.

Kaouane N, Porte Y, Vallée M, Brayda-Bruno L, Mons N, Calandreau L et al (2012). Glucocorticoids can induce PTSDlike memory impairments in mice. Science 335: 1510-1513.

Kjelstrup KG, Tuvnes FA, Steffenach HA, Murison R, Moser EI, Moser MB (2002). Reduced fear expression after lesions of the ventral hippocampus. Proc Natl Acad Sci USA 99: 1082510830 .

Laxmi TR, Stork O, Pape HC (2003). Generalisation of conditioned fear and its behavioural expression in mice. Behav Brain Res 145: 89-98.

Liotta A, Caliskan G, ul-Haq R, Hollnagel JO, Rösler A, Heinemann $\mathrm{U}$ et al (2011). Partial disinhibition is required for transition of stimulus-induced sharp wave-ripple complexes into recurrent epileptiform discharges in rat hippocampal slices. J Neurophysiol 105: 8172-8187.

Lu CB, Jefferys JG, Toescu EC, Vreugdenhil M (2011). In vitro hippocampal gamma oscillation power as an index of in vivo CA3 gamma oscillation strength and spatial reference memory. Neurobiol Learn Mem 95: 221-230.

Maggio N, Segal M (2007). Striking variations in corticosteroid modulation of long-term potentiation along the septotemporal axis of the hippocampus. J Neurosci 27: 5757-5765.

Maggio N, Segal M (2009). Differential corticosteroid modulation of inhibitory synaptic currents in the dorsal and ventral hippocampus. J Neurosci 29: 2857-2866.

Maren S, Holt WG (2004). Hippocampus and Pavlovian fear conditioning in rats: muscimol infusions into the ventral, but not dorsal, hippocampus impair the acquisition of conditional freezing to an auditory conditional stimulus. Behav Neurosci 118: $97-110$.

McGaugh JL (2004). The amygdala modulates the consolidation of memories of emotionally arousing experiences. Annu Rev Neurosci 27: 1-28.

Mitra R, Sapolsky RM (2008). Acute corticosterone treatment is sufficient to induce anxiety and amygdaloid dendritic hypertrophy. Proc Natl Acad Sci USA 105: 5573-5578.

Montgomery SM, Buzsáki G (2007). Gamma oscillations dynamically couple CA3 and CA1 region during memory task performance. Proc Natl Acad Sci USA 104: 14495-15000.

Olson VG, Rockett HR, Reh RK, Redila VA, Tran PM, Venkov HA et al (2011). The role of norepinephrine in differential response to stress in an animal model of posttraumatic stress disorder. Biol Psychiatry 70: 441-448.

Orchinik M, Caroll SS, Li Y, McEwen BS, Weiland NG (2001). Heterogeneity of hippocampal GABAA receptors: regulation by corticosterone. J Neurosci 21: 330-339.

Paxinos G, Franklin KBJ (2001). The Mouse Brain in Stereotaxic Coordinates, 2nd edn. Academic Press: San Diego.

Pitkänen A, Pikkarainen M, Nurminen N, Ylinen A (2000). Reciprocal connections between the amygdala and the hippocampal formation, perirhinal cortex, and postrhinal cortex in rat. A review. Ann N Y Acad Sci 911: 369-391.

Quirk GJ, Paré D, Richardson R, Herry C, Monfils MH, Schiller D et al (2010). Erasing fear memories with extinction training. J Neurosci 30: 14993-14997.

Radulovic J, Kammermeier J, Spiess J (1998). Generalization of fear responses in $\mathrm{C} 57 \mathrm{BL} / 6 \mathrm{~N}$ mice subjected to one-trial foreground contextual fear conditioning. Behav Brain Res 95: 179-189.

Rehberg K, Bergado-Acosta JR, Koch JC, Stork O (2010). Disruption of fear memory consolidation and reconsolidation by actin filament arrest in the basolateral amygdala. Neurobiol Learn Mem 94: 117-126.

Rodriguez-Ortiz CJ, Bermúdez-Rattoni F (2007). Memory reconsolidation or updating consolidation? In: Bermúdez-Rattoni $\mathrm{F}$ 
(ed.) Neural Plasticity and Memory: From Genes to Brain Imaging. CRC Press: Boca Raton, chapter 11.

Rudy JW, Matus-Amat P (2005). The ventral hippocampus supports a memory representation of context and contextual fear conditioning: implications for a unitary function of the hippocampus. Behav Neurosci 119: 154-163.

Schwabe L, Joels M, Roozendaal B, Oitzl MS (2012). Stress effects on memory: an update and integration. Neurosci Biobehav Rev 36: $1740-1749$.

Schelling G, Roozendaal B, De Quervain DJ (2004). Can posttraumatic stress disorder be prevented with glucocorticoids? Ann N Y Acad Sci 1032: 158-166.

Schiller D, Monfils MH, Raio CM, Johnson DC, Ledoux JE, Phelps EA (2010). Preventing the return of fear in humans using reconsolidation update mechanisms. Nature 463: 49-53.

Siegmund A, Wotjak CT (2006). Toward an animal model of posttraumatic stress disorder. Ann N Y Acad Sci 1071: 324-334.

Siegmund A, Köster L, Meves AM, Plag J, Stoy M, Ströhle A (2011). Stress hormones during flooding therapy and their relationship to therapy outcome in patients with panic disorder and agoraphobia. J Psychiatr Res 45: 339-346.
Soravia LM, Heinrichs M, Aerni A, Maroni C, Schelling G, Ehlert U et al (2006). Glucocorticoids reduce phobic fear in humans. Proc Natl Acad Sci USA 103: 5585-5590.

Stein DJ, Matsunaga H (2006). Specific phobia: a disorder of fear conditioning and extinction. CNS Spectr 11: 248-251.

Stranahan AM, Lee K, Mattson MP (2008). Contributions of impaired hippocampal plasticity and neurodegeneration to agerelated deficits in hormonal pulsatility. Ageing Res Rev 7: 164176.

Ströhle A, Holsboer F (2003). Stress responsive neurohormones in depression and anxiety. Pharmacopsychiatry 3: S207-S214.

Trivedi MA, Coover GD (2004). Lesions of the ventral hippocampus, but not the dorsal hippocampus, impair conditioned fear expression and inhibitory avoidance on the elevated T-maze. Neurobiol Learn Mem 81: 172-184.

Wang XY, Zhao M, Ghitza UE, Li YQ, Lu L (2008). Stress impairs reconsolidation of drug memory via glucocorticoid receptors in the basolateral amygdala. J Neurosci 28: 5602-5610.

Weiss EK, Krupka N, Bähner F, Both M, Draguhn A (2008). Fast effects of glucocorticoids on memory-related network oscillations in mouse hippocampus. J Neuroendocrinology 20: 549-557.

Supplementary Information accompanies the paper on the Neuropsychopharmacology website (http://www.nature.com/npp) 